\title{
An Inelastic Theoretical Model regarding the Load-Carrying Capacity of PSL Bending Component
}

\author{
Baolu Sheng $\mathbb{D}^{1,2}$ Yuling Bian, ${ }^{3}$ Dong He, ${ }^{4}$ and Aiping Zhou ${ }^{1,2}$ \\ ${ }^{1}$ National Engineering Research Center of Biomaterials, Nanjing Forestry University, 159 Longpan Rd., Nanjing 210037, China \\ ${ }^{2}$ School of Civil Engineering, Nanjing Forestry University, Nanjing 210037, China \\ ${ }^{3}$ Wuxi Vocational Institute of Commerce, 809 Qianhu Rd., Wuxi 214153, China \\ ${ }^{4}$ Dongfang Turbine Co.,Ltd., 666 Jinshajiang Rd., Deyang City, Sichuan Province 618000, China \\ Correspondence should be addressed to Baolu Sheng; baolu52520@163.com
}

Received 13 November 2020; Revised 2 December 2020; Accepted 27 February 2021; Published 11 March 2021

Academic Editor: Musa Adamu

Copyright ( $\odot 2021$ Baolu Sheng et al. This is an open access article distributed under the Creative Commons Attribution License, which permits unrestricted use, distribution, and reproduction in any medium, provided the original work is properly cited.

\begin{abstract}
Parallel strand lumber (PSL) is an attractive structural wood composite which may have prospective use in building constructions. Conducting nonlinear analysis for the bending of PSL beams is a critical step in the determination of ultimate strength and deflection of them, which is an essential requirement of the building design philosophy based on probability of ultimate state. For the purposes of this article, an inelastic theoretical model regarding the load-carrying capacity of the PSL bending component has been developed. Based on the uniaxial loading tests, the stress-strain behaviors of PSL composite in the grain direction were measured. 4-point bending experiments were also performed in this study to investigate the failure mechanism of the PSL components. The results show that the tensile stress-strain relationship of PSL materials in the grain direction remains linear until breaking, while the compressive stress-strain relationship exhibits nonlinear characteristics once the compressive stress exceeds the proportional limit, which can be expressed by a quadratic polynomial. The failure mode of the PSL beam can be summarized that the fibres in the top of the broken section were buckling and those in the bottom of the section were broken when failure occurred. Significant nonlinear behavior was exhibited based on the load-deflection curves of the PSL beams. To predict the nonlinear bending performance of the PSL beams, a theoretical model that could consider the nonlinear stress-strain relations of PSL and predict the damage modes of the PSL beams was developed. Well agreements can be observed between the results of calculations and experiments.
\end{abstract}

\section{Introduction}

PSL is a wood-based composite material with outstanding mechanical properties for construction. It is fabricated by gluing raw wood strands together along the grain direction under high pressure and microwave heat, which are often a by-product during the plywood manufacturing process [1]. After this industrial production process, the defects of raw wood will be eliminated. The consistent properties and fire resistance of PSL are superior to those of raw wood [2]. Thus, PSL has sufficient strength and rigidity [3] that makes PSL material very well suitable as beams and columns for crossand large-span building structure and for high-rise buildings [2]. In addition, due to the development of the energy- efficient building and green building initiatives, PSL has been becoming an extensively potential prospective structural material for building constructions.

It is a vital work for safeguard design to precisely evaluate the strength and deflection of the structural members in the condition of strength limit state. However, the structure and mechanical properties of PSL have a strong orientation. As a matter of fact, PSL is a natural oriented fiber-reinforced composite. The approach of strength theory and mechanical model proposed by classical theories to analyze the behaviors of the component made of homogeneous or isotropic materials cannot be suitable to conduct the inelastic analysis for PSL structural members. Additionally, linear principles are currently employed in design code for wood 
buildings to predict the load-carrying capacity and deflection in the strength limit state as the nonlinear behaviors of wood or wood composites have not been well modelled [2]. Unfortunately, considerable researchers have found that almost all engineered wood composites or raw wood materials, including PSL, have strong nonlinear characteristics in the stress-strain relationship under compression parallel to grain [4-6]. Wood-based structure members are actually displaying strong nonlinear performances when approaching the strength limit state. Accordingly, there are certain errors in the results inevitably in the calculation of the ultimate bearing capacity and the ultimate deformations of structure members by the use of the linear elastic principle. Therefore, to well understand the engineering behaviors of PSL composite, such as inelastic analysis and failure mechanisms, is the cornerstone of the development of wooden construction.

Some analytical or numerical models have been proposed to evaluate the nonlinear performances of wood or wood composite structure members. Naghipour et al. [7] developed a theoretical model to investigate the nonlinear behaviors of reinforced wood-plastic composite (WPC) beams wrapped with glass and carbon fiber-reinforced polymers. Nonlinear characteristics of WPC composite in the direction of tension and compression were considered by using an exponential function. Borri et al. [8] proposed a numerical procedure to study the performances of wood beams reinforced by using CFRP sheets. The bilinear model was adopted to simulate the elastic-plastic behavior in compression of wood material and linear elastic one in tension. Another model to investigate the flexural performances of CFRP retrofit wood beams was reported by Li et al. [9]. A quadratic curve was adopted to model the compressive nonlinearity of wood materials, and the function of the curve was obtained by data fitting for experiments. Actually, it is commonly accepted that the stress-strain relationship of raw wood materials in the grain direction is linear for tensile stress and nonlinear for compressive stress. Some mathematical models have been proposed for simulating this nonlinearity [6, 10-12]. Huang et al. [13] proposed an inelastic model of engineered bamboo bending components by considering the difference between tensile moduli and compressive moduli. The theoretical model, of which the nonlinearity of stress-strain relationships was considered, to predict the deflection, stress and strain, and ultimate bearing capacity of wood-based composite structural components in the strength limit state, however, is not available up to now.

In this paper, considering nonlinear behaviors of material, a theoretical model to predict the bending bearing capacity of PSL beams was developed and the ultimate deformation of the beam in midspan was also given by assuming a fictitious plastic hinge at the critical cross section. Firstly, mathematical formulas for describing the stressstrain relationships of PSL composite along the grain were proposed based on tests. Secondly, to investigate the failure mode and the failure mechanism of PSL beams, four-point bending tests were performed. A novel model, which takes the nonlinear stress-strain relationship of PSL composite into account, to predict the responses during all service periods from loading to failure for PSL bending members was developed at last. Well agreements can be observed between the results of calculations and experiments.

\section{Stress-Strain Relationship along Parallel to Grain Direction}

The stress-strain curve was obtained by experiments. Test materials were offered by FPInnovations, Vancouver, Canada. Tests were carried out in the laboratory of FPInnovations, Vancouver, Canada. Compressive test along the grain direction was referred to ASTM D143-09 [14]. Prisms with dimensions of $50 \mathrm{~mm} \times 50 \mathrm{~mm} \times 200 \mathrm{~mm}$ were adopted as specimens for tests. 22 specimens were tested. The loading direction should be perpendicular to both ends of the compressive specimen and parallel to fiber orientation. An axial compression test can use spherical hinge bearing to reallocate load traverse distribution so that the load could be evenly acted on the whole cross section passing through the roller. The loading procedure is controlled by the displacement method at a rate of motion of a movable crosshead of $0.6 \mathrm{~mm} / \mathrm{min}$. Longitudinal strain at the middle of the specimen was measured by strainometer. Specimens for the tensile test were also designed referring to ASTM 143-09 [13], and the dimensions of them (30 samples) are shown in Figure 1. Two ends of the tensile specimen are, respectively, clamped in the upper clamping head and the lower clamping head. The axis of the two clamping heads tends towards the same direction of the grain. The loading procedure is controlled by the displacement method at a rate of motion of a movable crosshead of $1 \mathrm{~mm} / \mathrm{min}$. A strainometer was fastened in the middle of the specimen to measure the strain over $50 \mathrm{~mm}$ length there. It was found that the curve of stress-strain relationships remains linear from loading to breaking when it was tensile failure. For the compressive properties, however, the stress varies linearly with the strain before the proportional point while becomes nonlinear when the stress exceeds the point.

The curve of uniaxial stress-strain relationships of PSL composites along the grain can be divided into three stages, i.e., first, the linear elasticity in tension, the linear elasticity in compression, and the nonlinear in compression, as shown in Figure 2. The nonlinear segment can be simulated via a second-order polynomial. Hence, the uniaxial longitudinal stressstrain relationships of the PSB composites can be represented as a piecewise function as shown in the following equation:

$$
\sigma(\varepsilon)= \begin{cases}l_{1}\left(\varepsilon+l_{2}\right)^{2}+l_{3}, & \varepsilon_{c e} \leq \varepsilon \leq \varepsilon_{c u}, \\ E \varepsilon, & -\varepsilon_{t u} \leq \varepsilon \leq \varepsilon_{c e},\end{cases}
$$

where $E$ is the elastic modulus of PSL material along parallel to grain direction (MPa). $\sigma_{c u}, \sigma_{c e}$, and $\sigma_{t u}$ are the stress at maximum compressive loading, proportional compressive limit, and ultimate tensile limit, respectively. $\varepsilon_{t u}$ and $\varepsilon_{c u}$ are the maximum strain in the tensile and compressive loading, respectively $(\mu \varepsilon)$, and $\varepsilon_{c e}$ is the compressive strain at the point of proportional limit $(\mu \varepsilon) . l_{1}, l_{2}$, and $l_{3}$ are coefficients.

Considering the continuum and compatible conditions of the stress-strain curves, the coefficients can be expressed as follows: 


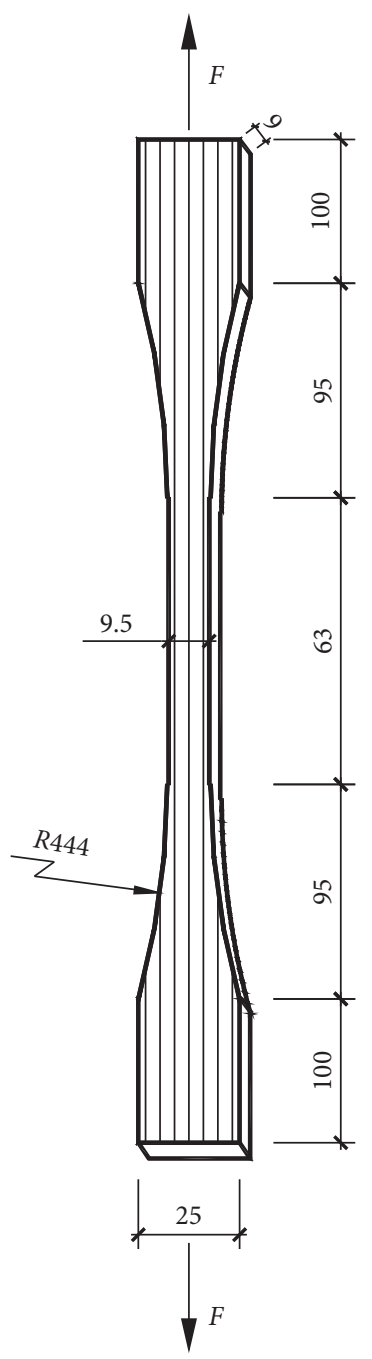

FIgURE 1: Dimension of tensile specimens (unit: $\mathrm{mm}$ ).

$$
\begin{aligned}
\sigma\left(\varepsilon_{c e}\right) & =l_{1}\left(\varepsilon_{c e}^{2}+2 l_{2} \varepsilon_{c e}+l_{2}^{2}\right)+l_{3}=\sigma_{c e}, \\
\sigma\left(\varepsilon_{c u}\right) & =l_{1}\left(\varepsilon_{c u}^{2}+2 l_{2} \varepsilon_{c u}+l_{2}^{2}\right)+l_{3}=\sigma_{c u}, \\
\frac{\mathrm{d} \sigma\left(\varepsilon_{c u}\right)}{\mathrm{d} \varepsilon} & =2 l_{1}\left(\varepsilon_{c u}+l_{2}\right)=0 .
\end{aligned}
$$

According to equations $(2 a)-(2 c)$, the coefficients can be obtained as follows:

$$
\begin{aligned}
& l_{1}=-\frac{\sigma_{c u}-\sigma_{c e}}{\left(\varepsilon_{c u}-\varepsilon_{c e}\right)^{2}}, \\
& l_{2}=-\varepsilon_{c u}, \\
& l_{3}=\sigma_{c u},
\end{aligned}
$$

Figure 2 illustrates the stress-strain relationships obtained by analyzing the test results. Table 1 gives the test results.

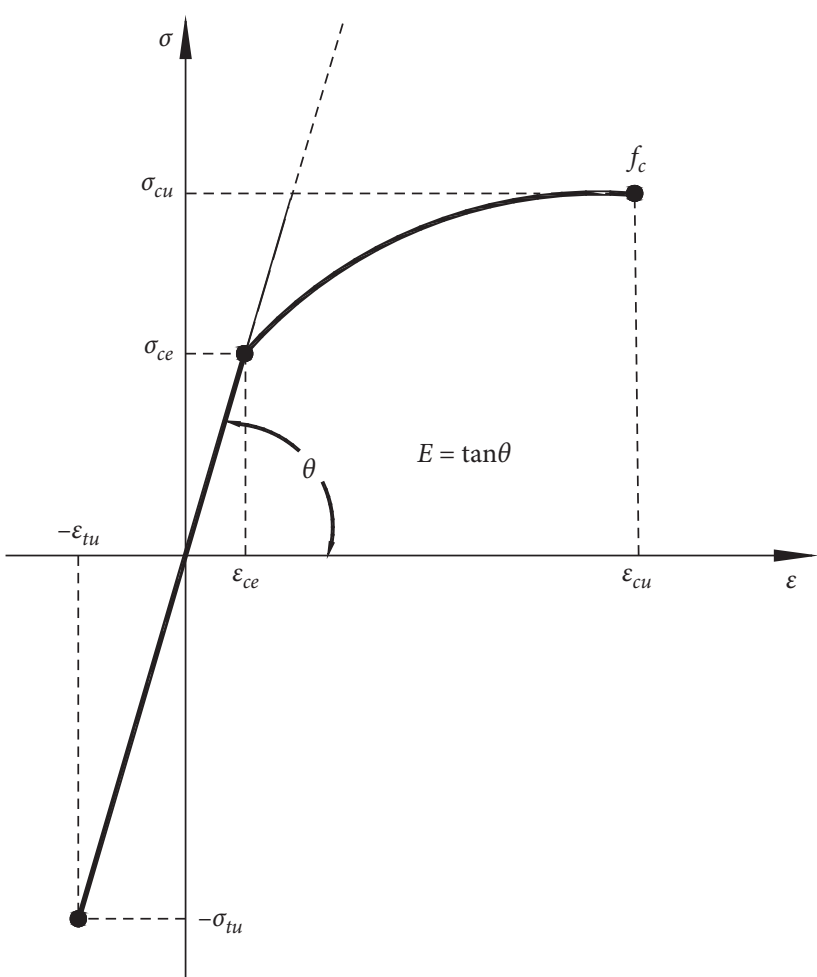

FIGURE 2: Uniaxial stress-strain relationships.

\section{Analytical Model}

3.1. Load-Carrying Capacity. The methodology for analyzing the nonlinear bending of the PSL beam in this research is based on Euler's beam theory and Huang's method [13, 15-17]. Fibres above and under the neutral axis were longitudinally compressed and tensioned, respectively, in case the beam was bent. The following assumptions were adopted: (1) cross section of the PSL beam above and under the neutral axis remains linear plane before and after loading; (2) the fibres in the top of the section reached maximum compressive stress and those in the bottom of the section reached ultimate tensile stress when the beam failed. Based on the above assumptions, the strain and stress diagram over the damage section shows a linear relationship; however, the distribution of compressive stress possessed nonlinear characteristics when stresses are beyond the compressive proportional limit, as shown in Figure 3. Thus, the stress distribution of the critical cross section of beam in moment span may be divided into three zones along the height of the PSL beam, i.e., plastic compressive zone (PCZ), elastic compressive zone (ECZ), and tensile zone (TZ). The upper part of the beam cross section is the PCZ, in which the stresses of the fibres in the upper outmost surface are equal to the ultimate compressive strength, $\sigma_{c u}$, when the beam fails. The area between the PCZ and the neutral axis is ECZ, in which the stresses and the strains of the fibres are equal to the compressive proportional limit, $\sigma_{c e}$ and $\varepsilon_{c e}$, respectively, in the interface surface between PCZ and TCZ. The area under the neutral axis is the TZ, in which the stresses and strains of the fibres in the lower outmost surface are equal to 
TABLE 1: Mechanical parameters of the PSL specimen.

\begin{tabular}{lccccc}
\hline \multirow{2}{*}{ Parameters } & \multicolumn{2}{c}{ Proportional limit } & \multicolumn{2}{c}{ Ultimate limit } & \multirow{2}{*}{ Modulus $(\mathrm{MPa})$} \\
& Strain $(\mu \varepsilon)$ & Stress $(\mathrm{MPa})$ & Strain $(\mu \varepsilon)$ & Stress $(\mathrm{MPa})$ & 80.0 \\
Tension & - & - & 1200 & 62.5 & 1650 \\
Compression & 2800 & 55.0 & 5700 & \\
\hline
\end{tabular}

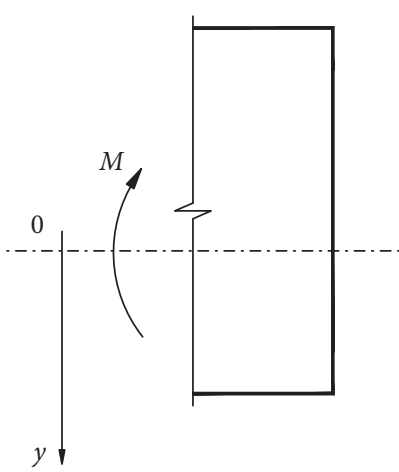

(a)

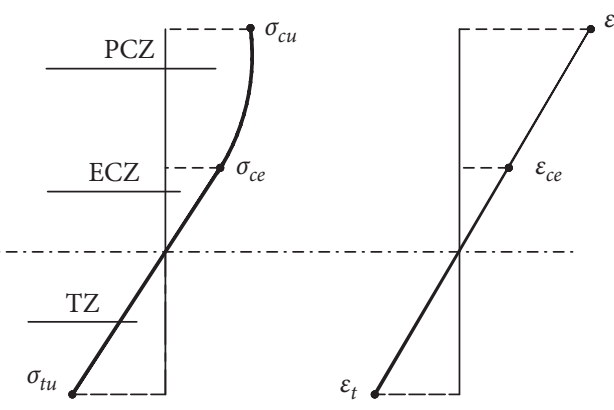

(b)

FIgURE 3: Diagrams of the stress and strain over the moment section. (a) stress diagram and (b) strain diagram.

the ultimate tensile strength, $\sigma_{t u}$ and $\varepsilon_{t u}$, respectively, when the beam broken. Thus, the force equilibrium in the longitudinal direction of the beam over the section yields

$$
b \int_{-\left(h_{p}+h_{c e}\right)}^{h_{t}} \sigma(y) \mathrm{d} y=0 .
$$

where $b$ is the width of the section. Because the compressive damage mechanism is very complex, it is impossible to functionally describe the actual stress distribution over the depth of the plastic zone.

Therefore, to exactly calculate the actual nonlinear stress distribution by using equation (4) is not practical. However, the actual nonlinear stress distribution may be approximately evaluated via equation (4) since the stress distribution over the depth of the plastic zone may be prescribed in accordance with the first term of equation (1) and the stress of outside fiber of the plastic zone is $\varepsilon_{c u}$. Thus, the stress with respect to the coordinate, $y$, over the depth of the plastic zone may be expressed as

$$
\varepsilon(y)=-\frac{\varepsilon_{c u}-\varepsilon_{c e}}{h_{p}} y,
$$

in which $-\left(h_{c e}+h_{p}\right) \leq y \leq-h_{c e}$ should be satisfied. Substituting for $\mathcal{E}(y)$ from equation (5) and for coefficients $l_{1}, l_{2}$, and $l_{3}$ from equations (3a)-(3c) into the first term of equation (1), the stress with respect to coordinate, $y$, over the depth of plastic zone, the actual nonlinear stress distribution can be obtained.

Therefore, the stress distribution with respect to the coordinate $y$ over the damaged cross section can be expressed as

$$
\sigma(y)= \begin{cases}l_{1}\left(s y+l_{2}\right)^{2}+l_{3}, & -\left(h_{c e}+h_{p}\right) \leq y \leq-h_{c e} \\ E s|y|, & -h_{c e} \leq y \leq h_{t}\end{cases}
$$

in which $s$ is the curvature of the beam at moment section. $h_{p}, h_{c e}$, and $h_{t}$ are the depths of plastic, compressive, and tensile zones, respectively. Referring to Figure 3(b) and taking the geometrical relationships into account lead to

$$
\begin{aligned}
h_{t} \varepsilon_{c e} & =\varepsilon_{t} h_{c e}, \\
\sigma_{t} & =E \varepsilon_{t} \text { and } \sigma_{c e}=E \varepsilon_{c e},
\end{aligned}
$$

where $\sigma_{t}$ is the tensile stress of the fibres in the outside of the tensile zone. Substituting for $\sigma(y)$ from equation (6) gives

$$
\int_{-\left(h_{p}+h_{c e}\right)}^{-h_{c e}}\left[l_{1}\left(s y+l_{2}\right)^{2}+l_{3}\right] \mathrm{d} y+\int_{-h_{c e}}^{h_{t}} E s|y| \mathrm{d} y=0,
$$

where

$$
s=-\frac{\varepsilon_{c u}-\varepsilon_{c e}}{h_{p}} .
$$

Also, taking the geometric relations of the zones over the section into account and referring to Figure 3(b) lead to

$$
h_{p}+h_{c e}+h_{t}=h \text {, }
$$

where $h$ is the depth of the section. From equations (7), (8), and (10), each depth of plastic, elastic compressive, and tensile zones can be obtained as follows:

$$
\begin{aligned}
& h_{p}=\left(\sigma_{c e}^{2}-\sigma_{t}^{2}\right) h \beta, \\
& h_{c e}=2 \sigma_{c e} h\left[\frac{l_{1}}{3}\left(\varepsilon_{c u}^{2}+\varepsilon_{c u} \varepsilon_{c e}+\varepsilon_{c e}^{2}\right)+\frac{l_{2}}{2}\left(\varepsilon_{c u}+\varepsilon_{c e}\right)+l_{3}\right] \beta,
\end{aligned}
$$

$$
h_{t}=2 \sigma_{t} h\left[\frac{l_{1}}{3}\left(\varepsilon_{c u}^{2}+\varepsilon_{c u} \varepsilon_{c e}+\varepsilon_{c e}^{2}\right)+\frac{l_{2}}{2}\left(\varepsilon_{c u}+\varepsilon_{c e}\right)+l_{3}\right] \beta,
$$


where

$$
\beta=\frac{1}{\left\{\sigma_{c e}^{2}+\left(2 / \sigma_{c e}\right)\left[\left(l_{1} / 3\right)\left(\varepsilon_{c u}^{2}+\varepsilon_{c u} \varepsilon_{c e}+\varepsilon_{c e}^{2}\right)+\left(l_{2} / 2\right)\left(\varepsilon_{c u}+\varepsilon_{c e}\right)+l_{3}\right]\left(\sigma_{c e}^{2}+\sigma_{c e} \sigma_{t}\right)-\sigma_{t}^{2}\right\}} .
$$

The moment at the section can be calculated by

$$
M=b \int_{-\left(h_{p}+h_{c e}\right)}^{h_{t}} \sigma(y) y \mathrm{~d} y .
$$

According to the plane assumption over the bending section cut, the strain at any point with respect to the coordinate, $y$, can be expressed as $\varepsilon(y)=s y$. Substituting $\varepsilon(y)$ into the first term of equation (6), the strain variation with respect to the coordinate, $y$, can be analytically obtained. Consequently, substituting for $\sigma(y)$ from equation (6) yields

$$
M=b\left\{\int_{-\left(h_{p}+h_{c e}\right)}^{-h_{c e}}\left[l_{1}\left(s y+l_{2}\right)^{2}+l_{3}\right] y \mathrm{~d} y+\int_{-h_{c e}}^{h_{t}} E s|y|^{2} \mathrm{~d} y\right\} .
$$

Finally, the function of the moment yielded by compressive force in the plastic zone can be expressed as

$$
\begin{aligned}
M= & \frac{1}{4} b l_{1} s^{2}\left[\left(h_{p}+h_{c e}\right)^{4}-h_{c e}^{4}\right]+\frac{1}{3} b l_{2} s\left[\left(h_{p}+h_{c e}\right)^{3}-h_{c e}^{3}\right] \\
& +\frac{1}{2} b l_{3}\left[\left(h_{p}+h_{c e}\right)^{2}-h_{c e}^{2}\right]+\frac{b E s}{3}\left(h_{c e}^{3}+h_{t}^{3}\right),
\end{aligned}
$$

where $s$ represents the curvature of the beam working in the nonlinear state. Considering the geometric relations between the strain, $\varepsilon$, and the curvature over the moment section, it can be obtained that $\sigma_{c e}=E s h_{c e}$. Deriving $h_{c e}$ from this equation and substituting $h_{c e}$ into equation (11b) yield

$$
s=\frac{1}{\left\{2 E h\left[\left(l_{1} / 3\right)\left(\varepsilon_{c u}^{2}+\varepsilon_{c u} \varepsilon_{c e}+\varepsilon_{c e}^{2}\right)+\left(l_{2} / 2\right)\left(\varepsilon_{c u}+\varepsilon_{c e}\right)+l_{3}\right] \beta\right\}} .
$$

In the case of critical condition, on which the stress on the top surface of the beam is just reached the proportional limit, $\sigma_{c e}$, and the whole section remains in the elastic state, the depth of the plastic zone of the section, $h_{p}=0$, and the plastic moment are zero. Therefore, it can be obtained from equation (11a) that

$$
\sigma_{t}^{2}-\sigma_{c e}^{2}=0 .
$$

Deriving $\sigma_{t}$ from this equation and substituting $\sigma_{t}$ into equations (11b) and (11c) yield the depths of compressive and tension zones on the critical condition, respectively, which are expressed as follows:

$$
h_{c e}=h_{t}=\frac{h}{2} \text {. }
$$

Let $s_{c}$ denote the curvature of the critical state, and the geometric relation between the strains and the curvature of the section leads to $\sigma_{c e}=E s_{c} h_{c e}$. Substituting $h_{c e}$ from equation (18) into this equation gives the curvature $s_{c}$, on the critical condition:

$$
s_{c}=\frac{2 \sigma_{c e}}{E h} .
$$

Let the plastic moment be zero, and substituting for $h_{c e}$ and $h_{t}$ from equation (18) into (13) and then replacing $s$ in equation (13) by $s_{c}$ from equation (19), the moment of the elastic limit of the beam, $M_{c}$, can be obtained:

$$
M_{c}=\frac{\sigma_{c e} b h^{2}}{6} \text {. }
$$

3.2. Deformation. Referring to Figure 4, the deflection analysis of the beam can be implemented by using the symmetrical half structure. Assume that the moment span is in nonlinear state and the curvature of it is $s$, while the shear span remains in the elastic state and the curvature of it is $s_{c}$. Hence, the differential equation governing the deformation of the beam can be expressed as

$$
\frac{\mathrm{d}^{2} y}{\mathrm{~d} x^{2}}= \begin{cases}-\frac{F x}{2 \mathrm{EI}}, & 0 \leq x \leq m, \\ -s, & m \leq x \leq \frac{l}{2} .\end{cases}
$$

The boundary and continuum conditions can be expressed as

$$
\begin{aligned}
y(0) & =0, \\
y^{+}(m) & =y^{-}(m), \\
\frac{\mathrm{d} y^{+}(m)}{\mathrm{d} x} & =\frac{\mathrm{d} y^{-}(m)}{\mathrm{d} x} \text { and } \frac{\mathrm{d} y(l / 2)}{\mathrm{d} x}=0 .
\end{aligned}
$$

From equations (21) and (22), the deformation equations of the beam with respect to the coordinate $x$ can be obtained, which were expressed as follows:

$$
y= \begin{cases}-\frac{F}{4 \mathrm{EI}}\left[\frac{1}{3} x^{3}+(m-l) m x\right], & 0 \leq x \leq m, \\ -\frac{1}{2} s\left(x^{2}-l x+\frac{1}{3} m^{2}\right), & m \leq x \leq \frac{l}{2} .\end{cases}
$$




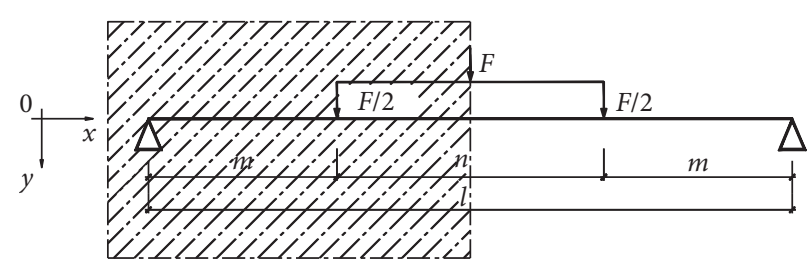

Figure 4: Deflection analysis model.

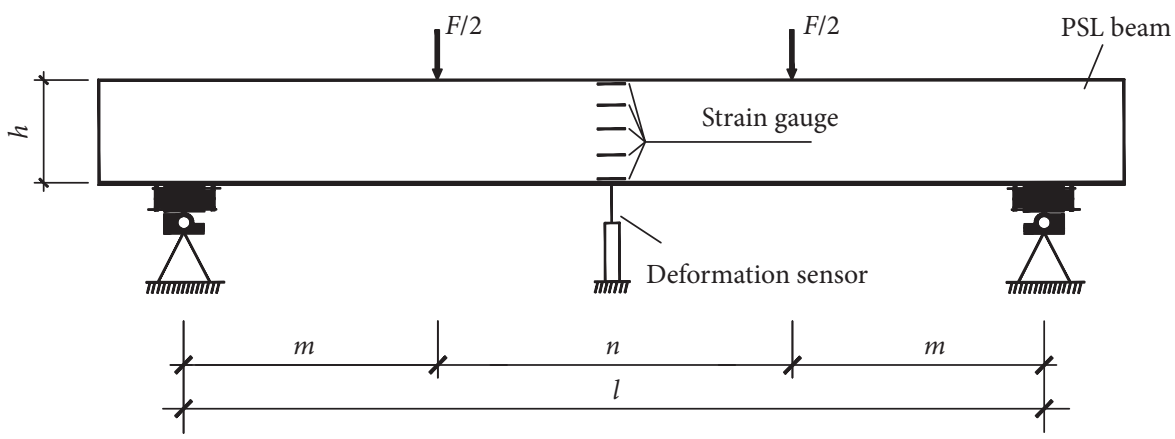

Figure 5: 4-point bending test.

where $I=\left(b h^{3} / 12\right)$. If the moment span works in the elastic state, considering the continuum condition at the section $x=m$ yields the curvature of this segment

$$
s_{m}=\frac{F m}{2 \mathrm{EI}} \text {. }
$$

Replacing $s$ in equation (23) with $s_{\mathrm{m}}$ in equation (24) gives the curvature equation of the beam

$$
y= \begin{cases}-\frac{F}{4 \mathrm{EI}}\left[\frac{1}{3} x^{3}+(m-l) m x\right], & 0 \leq x \leq m, \\ -\frac{F m}{4 \mathrm{EI}}\left(x^{2}-l x+\frac{1}{3} m^{2}\right), & m \leq x \leq \frac{l}{2} .\end{cases}
$$

According to Huang's method [18], the deformation at midspan of the PSL beam can be expressed as

$$
v=-\frac{F m}{4 \mathrm{EI}}\left(\frac{1}{3} m^{2}-\frac{1}{4} l^{2}\right)+\frac{1}{4} \varepsilon_{t} h\left(\frac{1}{h_{t}}-\frac{2}{h}\right),
$$

where $\nu$ is the deformation at midspan of the PSL beam.

\section{Test Validation}

In order to validate the analytical model developed above, load-carrying capacities and deformations of three PSL beams were analyzed by the model above and by experiments. 4-point bending test was adopted to investigate the bending performances of the PSL beam. Test method was referred to ASTM D198-09 [19]. The section dimensions of the samples are $60 \mathrm{~mm}$ in width and $90 \mathrm{~mm}$ in depth. The test span is $1660 \mathrm{~mm}$. Beams were supported by a pair of metal roller which has sufficient stiffness and provides unrestricted longitudinal deformation and rotation of beam at reactions due to

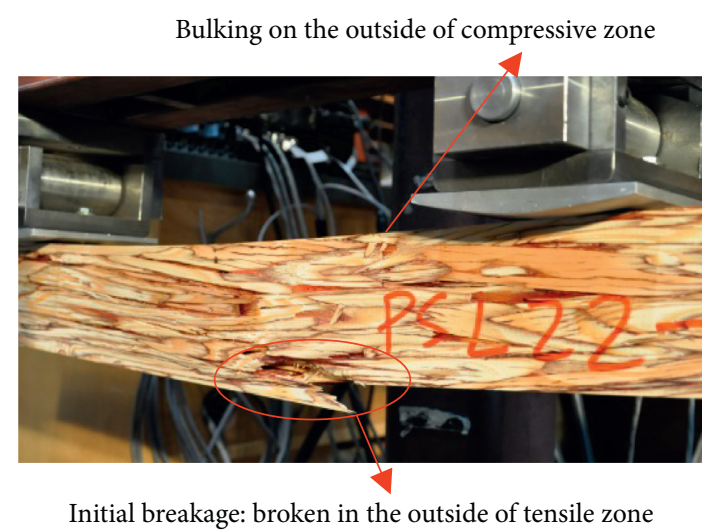

FIgURE 6: Damage mode of the PSL beam.

loading. The total load on the beam was symmetrically and monotonously applied at two points equidistant from the reactions through a pair of blocks, which are extending entirely across the beam width and may rotate the axis perpendicular to the span without restraint. The test setup is shown in Figure 5. A deformation sensor was installed under the middle span of the beam to measure the vertical displacement there. The total load of the beam and the vertical displacement in the middle span were simultaneously recorded. The failure mode is shown in Figure 6. Local buckling at the top and tensile broken at the bottom of the beam can be observed. This is in agreement with the assumption in Section 3. Figure 7 compares the load-deflection curves between experimental results and calculations using the theoretical model, of which the mechanical parameters of PSL were taken from Table 1. Well agreement can be observed. This implies that the model developed in this paper for analyzing the bending of PSL beams is feasible. 


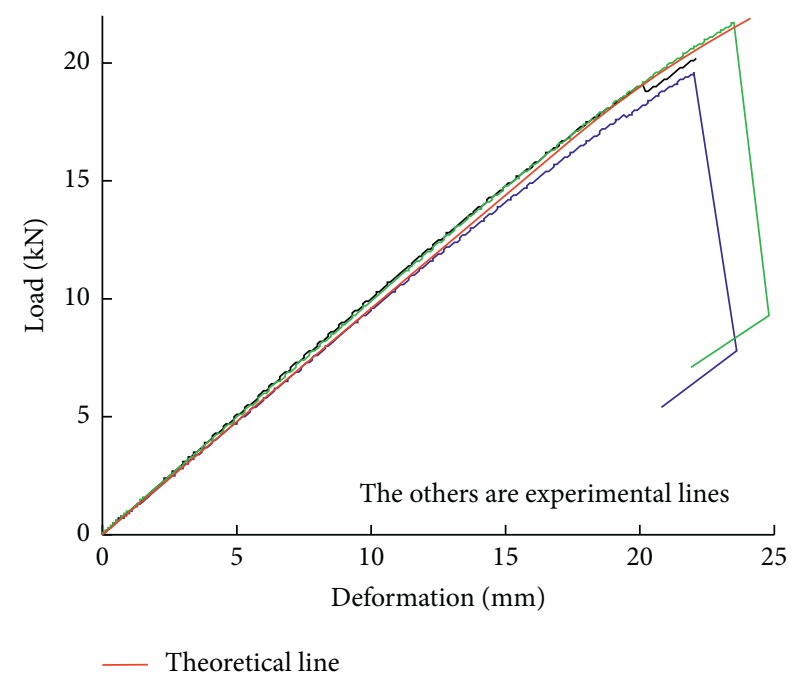

Figure 7: Comparison of the load-deflection curves of PSL beams obtained by test and analysis.

\section{Conclusions}

Based on the theories of mechanics of composite, PSL was treated as a transversely isotropic composite, and this paper aimed at studying the stress-strain relationships, the failure mechanism, and the nonlinear flexural of PSL beams through the experiments and theoretical analysis. The main contents and results can be concluded as follows:

(1) The uniaxial tensile and compressive properties in parallel to grain direction were studied by experiments. Failure mechanisms and the stress-strain relationship of each stress state were investigated. It was found that the tensile failure of PSL in parallel to grain direction presents the progressive process and higher strength, and the tensile stress-strain relationship exhibits linear behavior. The compressive strength of the material shows lower strength and brittle behavior, and then the compressive stressstrain relationship exhibits nonlinear characteristics.

(2) 4-point bending tests for PSL beams were carried out to investigate the flexural behaviors and the damage mechanism of them. 3 stages of bending failure of PSL beams under pure bending loading can be observed. The first is the perfect elastic bending stage, and the beams were in the elastic state when the loading is less than the proportional limit. The second is the nonlinear hardening stage, i.e., when the loading exceeded the proportional limit, part of fibres in the compressive zone in the critical section came into a nonlinear state, the inelastic compressive zone was gradually expanding towards the neutral axis, and the stiffness of PSL beams was continuously degraded with the augment of loading. The last one is the failure stage, i.e., when the depth of the inelastic compressive zone reached the ultimate value, the fibres in the outer surface of the compressive zone were bulking and those in the outer surface of the tensile zone were broken. Hence, the PSL beam was failed.

(3) A theoretical model to evaluate the loading capacity and the deflection of PSL beams was proposed based on experimental studies and theoretical analysis. Well agreements were achieved between the results obtained by using the proposed model and those obtained by experiments.

\section{Data Availability}

The data used to support the findings of this study are available from the corresponding author upon request.

\section{Conflicts of Interest}

The authors declare that there are no conflicts of interest regarding the publication of this paper.

\section{Acknowledgments}

The research was supported by the National Science Fund of China (no. 51978338), the Priority Academic Program Development of Jiangsu Higher Education Institutions (PAPD), and the Doctorate Fellowship Foundation of Nanjing Forestry University.

\section{References}

[1] P. Sukontasukkul, F. Lam, and S. Mindess, "Fracture of parallel strand lumber (PSL) under impact loading," Materials and Structures, vol. 33, pp. 445-449, 2000.

[2] Canadian Wood Council, Wood Design Manual, Canadian Wood Council, Ottawa, Canada, 2010.

[3] S. R. Arwade, R. Winans, and P. L. Clouston, "Variability of the compressive strength of parallel strand lumber," Journal of Engineering Mechanics, vol. 136, no. 4, pp. 405-412, 2010.

[4] V. Yadama, M. P. Wolcott, and L. V. Smith, "Elastic properties of wood-strand composites with undulating strands," Composites Part A: Applied Science and Manufacturing, vol. 37, no. 3, pp. 385-392, 2006.

[5] P. L. Clouston and F. Lam, "A stochastic plasticity approach to strength modeling of strand-based wood composites," Composites Science and Technology, vol. 62, no. 10-11, pp. 1381-1395, 2002.

[6] X. Song and F. Lam, "Laterally braced wood beam-columns subjected to biaxial eccentric loading," Computers \& Structures, vol. 87, no. 17-18, pp. 1058-1066, 2009.

[7] M. Naghipour, M. Nematzadeh, and Q. Yahyazadeh, "Analytical and experimental study on flexural performance of WPC-FRP beams," Construction and Building Materials, vol. 25, no. 2, pp. 829-837, 2011.

[8] A. Borri, M. Corradi, and A. Grazini, "A method for flexural reinforcement of old wood beams with CFRP materials," Composites Part B: Engineering, vol. 36, no. 2, pp. 143-153, 2005.

[9] Y.-F. Li, Y.-M. Xie, and M.-J. Tsai, "Enhancement of the flexural performance of retrofitted wood beams using CFRP composite sheets," Construction and Building Materials, vol. 23, no. 1, pp. 411-422, 2009. 
[10] I. M. M. Bazan, "Ultimate bending strength of timber beams," Doctoral dissertation, Thesis presented to Nova Scotia Technological College, Halifax, Canada, 1980.

[11] M. R. O’Halloran, “Curvilinear stress strain relationship for wood in compression," Doctoral dissertation, Thesis presented to Colorado State University, Fort Collins, Colorado, 1973.

[12] A. Ylinen, "A method of determining the buckling stress and the required cross sectional area for centrally loaded straight columns in elastic and inelastic range," IABSA Publications, vol. 16, pp. 529-549, 1956.

[13] D. Huang, A. Zhou, and Y. Bian, "Experimental and analytical study on the nonlinear bending of parallel strand bamboo beams," Construction and Building Materials, vol. 44, pp. 585-592, 2013.

[14] American Society for Testing and Materials (ASTM), Standard Test Method for Small Clear Specimens for Timber: D143-09, American Society for Testing and Materials, West Conshohocken, PA, USA, 2009.

[15] A. Zhou, D. Huang, H. Li, and Y. Su, "Hybrid approach to determine the mechanical parameters of fibers and matrixes of bamboo," Construction and Building Materials, vol. 35, pp. 191-196, 2012.

[16] D. Huang, Y. Bian, A. Zhou, and B. Sheng, "Experimental study on stress-strain relationships and failure mechanisms of parallel strand bamboo made from phyllostachys," Construction and Building Materials, vol. 77, pp. 130-138, 2015.

[17] B. D. Zakic, "Inelastic bending of wood beams," in Proceedings of ASCE, vol. 99, no. ST10, pp. 2079-2095, 1974.

[18] Z. Huang, Z. Chen, D. Huang, and A. Zhou, "The ultimate load-carrying capacity and deformation of laminated bamboo hollow decks: experimental investigation and inelastic analysis," Construction and Building Materials, vol. 117, pp. 190-197, 2016.

[19] American Society for Testing and Materials (ASTM), Standard Test Method of Static Texts of Lumber in Structural Size: D198-09, American Society for Testing and Materials, West Conshohocken, PA, USA, 2009. 